\title{
Trivium
}

Revue franco-allemande de sciences humaines et sociales - Deutsch-französische Zeitschrift für Geistesund Sozialwissenschaften

31 | 2020

La culture politique de la République romaine

\section{Die politische Kultur der römischen Republik in der deutschen Forschung}

\section{Martin Jehne}

\section{OpenEdition \\ Journals}

Édition électronique

URL : https://journals.openedition.org/trivium/7246

DOI : $10.4000 /$ trivium.7246

ISBN : 1963-1820

ISSN : 1963-1820

Éditeur

Les éditions de la Maison des sciences de l'Homme

Référence électronique

Martin Jehne, „Die politische Kultur der römischen Republik in der deutschen Forschung“, Trivium [Online], 31 | 2020, online erschienen am 30 Juni 2020, abgerufen am 23 August 2021. URL: http:// journals.openedition.org/trivium/7246 ; DOI: https://doi.org/10.4000/trivium.7246

Ce document a été généré automatiquement le 23 août 2021.

\section{(c) (1) $(9$}

Les contenus des la revue Trivium sont mis à disposition selon les termes de la Licence Creative Commons Attribution - Pas d'Utilisation Commerciale - Pas de Modification 4.0 International. 


\title{
Die politische Kultur der römischen Republik in der deutschen Forschung
}

\author{
Martin Jehne
}

1 Am Anfang war Res publica amissa. ${ }^{1}$ Natürlich begann die deutsche Forschung zur römischen Republik nicht erst mit diesem herausragenden Werk Christian Meiers, und er selbst bezog durchaus wichtige Impulse vor allem von Theodor Mommsen, Matthias Gelzer, Hermann Strasburger und Alfred Heuß,2 deren Werke auch außerhalb Deutschlands geschätzt und breiter rezipiert wurden. ${ }^{3}$ Dennoch markiert Meiers Buch Res publica amissa einen Perspektivenwandel und damit einen Einschnitt, der bis heute nachwirkt. In seinem Bemühen, besser zu verstehen, warum denn die römische Republik untergegangen ist, konturierte Meier die politische Praxis neu. Auch er maß den - von Gelzer so benannten - »Nah- und Treuverhältnissen«, d. h. den Beziehungen zwischen Patronen und Clienten einerseits und den Solidaritäten der Familie und der Freundschaft auf der Ebene der sozial Gleichgestellten andererseits, eine große Bedeutung bei; aber er legte überzeugend dar, dass dieses System der Austauschbeziehungen sich wandelte und im 2. und 1. Jahrhundert v. Chr. keineswegs mehr in der Lage war, eine hohe Berechenbarkeit von Wahlen und anderen wichtigen Entscheidungen zu garantieren. So war die römische Politik von einem ständigen Bemühen durchzogen, in situativen Koalitionen die nötige Unterstützung zu organisieren. Die Politik verlangte also permanente Geschäftigkeit und hohe Beweglichkeit. Die stabileren Faktionen, die man früher aus den Verwandtschafts- und Austauschgemeinschaften herauszulesen pflegte, waren in der späten Republik nicht vorhanden - jedenfalls nicht in dem Umfang, der erforderlich gewesen wäre, um sich im konkreten Fall auf das Durchsetzungsvermögen der eigenen Parteiung verlassen zu können. Zugleich hatte die enorme römische Expansion massive Rückwirkungen auf die Institutionen, aber auch auf das Verhalten von Gruppen und Individuen. Da man gleichzeitig an den gewachsenen Verfahrensweisen und Machtverteilungen festhielt, wurde die Ordnung des Gemeinwesens massiv überdehnt - Meier spricht hier von der Extensivierung der res publica. Als eine Folge dieser Extensivierung beobachtete Meier 
ein in der Führungsschicht durchaus verbreitetes Unbehagen in Bezug auf die politischen Verhältnisse, ohne dass diese Negativsicht grundsätzlichere Reformideen oder gar einen stärkeren Veränderungswillen hervorgebracht hätte. Für diese Lage prägte Meier den neuen Begriff der "Krise ohne Alternative«, den er statt des von Mommsen herrührenden, von Heuß aber theoretisch fundierten Revolutionsbegriffs für die Lage der nachsullanischen Republik verwenden wollte. ${ }^{4}$

2 Meier hatte eine Reihe von Schülern, ohne selbst im verengenden Sinne Schule zu bilden, d. h.: Interessen und Zugriffsweisen seiner Schüler waren sehr unterschiedlich. Für die späte römische Republik sind zunächst Kurt Raaflaub und Hinnerk Bruhns zu nennen, die wesentliche Arbeiten über den Ausbruch des Bürgerkrieges und die Spaltung der Führungsschicht vorlegten, welche zweifellos von dem starken Interesse Meiers an Caesars Bedeutung für den Untergang der Republik angeregt waren. ${ }^{5}$ Rolf Rilinger untersuchte die Rolle des Wahlleiters und widerlegte gründlich die verbreitete Vorstellung, hier stünde der römischen Führungsschicht ein jederzeit einsetzbares Mittel zur Verfügung, einem unerwünschten Wahlergebnis entgegenzusteuern. ${ }^{6}$ Auch analysierte er die konkurrierenden Begriffe der »Revolution" und der "Krise ohne Alternative « und konnte dabei die Vorzüge der Meier'schen Neuschöpfung herausarbeiten. ${ }^{7}$ Hinnerk Bruhns griff in einem Artikel zur Geschichte des Krisenbegriffs einerseits weit über die Altertumswissenschaften hinaus, andererseits verdeutlichte er, dass die Anwendungen auf die Verhältnisse der späten römischen Republik oft wenig reflektiert sind, woraus die Bedeutung von Meiers Innovation umso klarer hervorgeht. ${ }^{8}$ Wilfried Nippel beschäftigte sich mit der öffentlichen Ordnung in Rom und zeigte überzeugend, wie ein traditionelles Normensystem, das nicht nur die Gebildeten, sondern auch die einfachen Bürger internalisiert hatten, dafür sorgte, dass trotz aller Konflikte einerseits die Rituale der politischen Kommunikation meistens störungsfrei abliefen, andererseits aber auch massive Konflikte nur selten in Gewalt umschlugen und schon gar nicht auf eine grundlegende Änderung der politischen Strukturen abzielten. Zudem konnte er auf dieser Basis auch deutlich machen, dass die plebs bei starken Normverletzungen durchaus spontan und eigenständig handlungsfähig war und nicht in all ihren Reaktionen von Rädelsführern aus der Oberschicht gesteuert wurde. ${ }^{9}$ Angeregt von Meiers Forschungen zur Republik rekonstruierte und analysierte Karl-Joachim Hölkeskamp die Formierungsphase der römischen Nobilität in der frühen und mittleren Republik und erhellte die Entstehung von Regeln und Strukturen, die dann in späteren Zeiten als unumstößliche Kernelemente der republikanischen Ordnung galten. ${ }^{10}$

3 Zu der Zeit, als Christian Meier sein neues Verständnis römischer Politik vorstellte, begann die Sozialgeschichte zum Schwerpunkt der Geschichtswissenschaften allgemein zu werden, und davon blieb die Alte Geschichte nicht unberührt. Überhaupt interessierte man sich vermehrt für diejenigen Gruppen, die nicht im Rampenlicht der Geschichte zu stehen pflegen. Bemerkenswert sind die Impulse, die Jochen Martin dieser Forschungsrichtung mit seiner Hinwendung zur Historischen Anthropologie verlieh (für die an der Universität Freiburg, wo Martin lange lehrte, ein breit interdisziplinär arbeitendes Institut gegründet wurde).${ }^{11} \mathrm{Er}$ forschte und koordinierte Forschung über Kindheit, die Stellung des Vaters allgemein in der Antike, über Männer- und Frauenrollen und überhaupt über die Familie und bemühte sich so, dem Kern einer im eigentlichen Sinne "Historischen" Anthropologie auf die Spur zu kommen: dem »Wandel des Beständigen «. ${ }^{12}$ Das Interesse an Familienstrukturen und 
ihrer gesellschaftlichen Bedeutung ist nach wie vor groß. ${ }^{13}$ Darüber hinaus geriet verstärkt die Sklaverei in den Blick der Forschung, für die es schon seit 1950 eine Forschungsstelle an der Akademie in Mainz gab, die prononciert deshalb gegründet worden war, um dem marxistischen Fokus auf die sog. Sklavenhaltergesellschaften etwas an die Seite zu stellen oder gar entgegenzusetzen..$^{14}$ Insgesamt beschäftigte sich die oft prosopographisch orientierte Sozialgeschichte der Zeit eher mit den Oberschichten der Kaiserzeit, für die mit den Inschriften ein umfangreicher Quellenbestand zur Verfügung stand, doch als Géza Alföldy 1976 seine Römische Sozialgeschichte herausbrachte, behandelte er selbstverständlich auch die römische Republik. ${ }^{15}$ Die lange wenigen Spezialisten überlassene Wirtschaftsgeschichte wurde nun auch für die Antike wieder vermehrt betrieben, wobei hier die Anregungen von Moses Finley zweifellos eine bedeutende Rolle spielten. ${ }^{16}$ In Deutschland war es vor allem Helmuth Schneider, der sich darum bemühte, wirtschaftsgeschichtliche Perspektiven auch für die römische Republik stärker in den Vordergrund zu rücken. ${ }^{17}$

4 Angesichts der langen staatsrechtlichen Tradition der Republikforschung in Deutschland ist es nicht verwunderlich, dass dies ein wichtiges Arbeitsfeld blieb. Der herausragende Vertreter dieser Richtung war Jochen Bleicken, der aber schon in seiner Dissertation über das Volkstribunat der mittleren Republik neben den eher staatsrechtlichen Elementen die politische Rolle dieses Amtes und seiner Inhaber ins Zentrum rückte, indem er die Bindung an den Senat und die weitgehend kooperative Haltung der Tribune in dieser Epoche als ein außerrechtliches Phänomen herausarbeitete. ${ }^{18}$ In seinem Werk über die römische Gesetzgebung sortierte er die Beschlüsse der römischen Volksversammlungen nach ihrem situationsgebundenen oder normativen Charakter auseinander und konzeptualisierte die Gesetzesflut der späten Republik als eine Versteifung, mit der die außerrechtlichen persönlichen Bindungen egalitärer und hierarchischer Art, der Kern der aristokratischen Ordnung, in ihrem Spannungsverhältnis zu entpersonalisierten Vorschriften vermehrt unter Druck gerieten. ${ }^{19}$ Die Gegenüberstellung von Rechts- und Sozialordnung hat Christian Meier jedoch kritisiert. ${ }^{20}$ Zudem hat Christoph Lundgreen mittlerweile mit seiner Unterscheidung zwischen Regeln und Prinzipien verdeutlicht, wie die römische Republik teilweise mit weichen Normen operierte, die im Einzelfall beiseitegeschoben werden konnten, ohne ihren normativen Charakter einzubüßen. ${ }^{21}$

5 In Frankreich erschienen im Jahre 1976 zwei grandiose Werke: Le métier de citoyen von Claude Nicolet und Le pain et le cirque von Paul Veyne. ${ }^{22}$ In beiden spielten die eigentümlichen Beziehungen zwischen Volk und Eliten in Rom eine zentrale Rolle, aber die Schwerpunkte und Herangehensweisen waren sehr unterschiedlich. Dennoch kann man, so glaube ich, die Perspektiven und Deutungsansätze als einen "communicative turn " in der Erforschung der römischen Geschichte bezeichnen. ${ }^{23}$ Die Rezeption in Deutschland war vorhanden, aber begrenzt, wobei Veynes Buch (wie auch andere) ins Deutsche übersetzt wurde und daher bessere Chancen hatte als Nicolets Werk, dessen amerikanische Version möglicherweise öfter benutzt wurde als das französische Original. Dem Austausch zwischen der französischen und der deutschen Forschung zur römischen Republik, der immer zu wünschen übrig ließ, halfen Jean-Michel David, Hinnerk Bruhns und Wilfried Nippel auf die Sprünge: Sie organisierten 1994 einen Kongress in Strasbourg, bei dem Beiträge zu Kernthemen der späten Republik vorgelegt wurden, die von einem Vertreter bzw. einer Vertreterin des jeweils anderen Landes 
kommentiert wurden. ${ }^{24}$ Differenzen wie Gemeinsamkeiten, vor allem aber auch Anknüpfungspunkte traten dabei deutlicher zutage.

6 Einen wesentlichen Impuls erhielt die Erforschung der römischen Republik allgemein und vielleicht besonders in Deutschland durch die Arbeiten von Fergus Millar seit 1984. Millar hatte sich zuvor vor allem mit der römischen Kaiserzeit und der Spätantike beschäftigt und mit dem systematischen Werk über den römischen Kaiser als Herrschaftsstruktur einen weiterhin zu den Grundlagenwerken gehörenden Klassiker verfasst. ${ }^{25}$ Jetzt wandte er sich der Republik zu und begründete in einer Reihe von Aufsätzen seine neue Kernthese, dass es sich bei der römischen Republik entgegen der gängigen Sichtweise, dass das politische System als aristokratisch bzw. oligarchisch einzustufen sei, im Kern um eine Demokratie gehandelt habe. ${ }^{26}$ Seine Einschätzung basiert auf den unbezweifelbaren Tatsachen, dass Politik in Rom öffentlich vor den Bürgern erläutert und plausibilisiert werden musste und dass die bindenden Entscheidungen in Volksversammlungen durch Abstimmung aller anwesenden Bürger getroffen wurden. Millars neue Deutung brachte Zustimmung und Widerspruch hervor, ${ }^{27}$ aber gerade für die Historiker/innen in Deutschland, deren Blick auf die römische Republik durch Meiers Res publica amissa inspiriert war, war diese Klassifikation wenig einleuchtend. Auf dem Deutschen Historikertag in Leipzig 1994 wurde dann von Egon Flaig, Karl-Joachim Hölkeskamp und mir eine Sektion mit dem Titel »Demokratie in Rom? Die Rolle des Volkes in der Politik der römischen Republik« abgehalten, deren Beiträge im nächsten Jahr als Buch publiziert wurden..$^{28}$ Die Kritik an den Einschätzungen Fergus Millars wurde hier vor allem mit den Kategorien und Konzepten der politischen Kulturforschung begründet, und seither rückte das Thema wie der Ansatz in der deutschen Forschung zur römischen Republik immer mehr in den Vordergrund..$^{29}$

7 Egon Flaig hatte schon in seinem Buch über die Usurpationen der frühen Kaiserzeit eine bestimmte Form des Umgangs mit dem römischen Volk in Versammlungen als Konsensritual bezeichnet. ${ }^{30}$ Damit bewegte er sich entlang der fundamentalen Unterscheidung der politischen Kulturforschung zwischen der Inhalts- und der Ausdrucksseite der Politik, die den Blick dafür öffnete, dass politische Systeme, die sich auf eine partizipierende Bürgergemeinschaft berufen, durchaus nicht so selten mit ritualisierten Kommunikationsakten in Versammlungen >des Volkes` operieren. Dabei bestätigen die Eliten dem Volk seine Bedeutung und das Volk den Eliten deren präformierte Entscheidungsempfehlungen. Wenn dazu dann noch - wie im republikanischen Rom - eine Struktur der Personenwahlen existiert, in der keine politischen Konzepte, sondern nur mehr oder weniger ehrenhafte Persönlichkeiten zur Wahl stehen, von deren Amtsführung man normalerweise keine relevanten Differenzen zu erwarten hatte, dann ist die Bedeutsamkeit der konkreten Wahlentscheidung ohnehin nicht sehr hoch anzusetzen. ${ }^{31}$ Auf diesen Ansätzen, deren Quellen KarlJoachim Hölkeskamp in seinem Beitrag zu dieser Trivium-Ausgabe noch einmal knapp zusammengefasst hat, ${ }^{32}$ bauten die Analysen des Funktionierens der römischen Republik in der Folgezeit auf. In dem Buch Ritualisierte Politik von 2003, in dem Egon Flaig seine Forschungen zur römischen Republik systematisch zusammengebunden hat, werden die verschiedenen Anregungen deutlich, die gerade französische Soziologen und Historiker der Republikforschung in Deutschland gegeben haben..$^{33}$ Unter den ritualisierten Formen der Kommunikation, die Flaig in seinem Buch analysiert hat, seien hier die zwingenden Gesten hervorgehoben - dem Trauergestus hat jetzt Christoph Degelmann eine eigene Monographie gewidmet. ${ }^{34}$ Jan Timmer hat gezeigt, 
wie weitreichend Konsenselemente in die Verfahren und Rituale im Senat eingebaut waren. ${ }^{35}$

8 Seit den späteren 1990er Jahren war die Frage nach der Rolle des römischen Volkes in der Politik der republikanischen Zeit ein zentraler Gegenstand der Republikforschung, national wie international. Während man in der englischsprachigen Forschung den Auffassungen von Millar zum Teil folgte, war man in Deutschland eher skeptisch. Ein wesentlicher Teil der Analysen fokussierte sich auf die contiones in ihrem doppelten Sinne als Versammlung und als Rede vor der Versammlung und damit auf die Performanz der Redner. ${ }^{36}$ Neue Bewegung kam in die Debatte, als Henrik Mouritsen seine These vorgestellt hatte, dass die römischen Volksversammlungen nicht nur deshalb nicht als demokratisches Element anzusehen seien, weil sie im Wesentlichen eine Zustimmungsfunktion ausübten, sondern auch deshalb, weil das Volk im Sinne der weniger gutgestellten Bürger gar nicht anwesend war. Unter den in den contiones präsenten Zuhörern habe man mit einer Dominanz von Parteigängern aus der leisure class zu rechnen. ${ }^{37}$ Gegen diese Theorie, die einen Gegenpol zu Millars Auffassungen bildet, regte sich ebenfalls Widerstand, unter anderem mit dem Argument, dass bescheidene ökonomische Verhältnisse in der Vormoderne keineswegs bedeuteten, dass solche Bürger nie Zeit fanden, sich politische Reden anzuhören - zumal es in den contiones üblich war, dass die Redner den Anwesenden schmeichelten und das Letztentscheidungsrecht des Volkes betonten. ${ }^{38}$ Doch gibt es auch eine gewisse Bewegung gegen eine Unterschätzung des Einflusses des einfachen Volkes und gegen die der Überschätzung der Kontrolle von oben. ${ }^{39}$ Als Teil der näheren Betrachtung der Kommunikationsformen zwischen Ober- und Unterschicht in der stadtrömischen Politik wurden auch die komplexen Wahlverfahren und die Wahlkampfaktivitäten der Kandidaten, einschließlich des ambitus (der als illegal aufgefassten Beeinflussung vor allem durch Geldzahlungen): vielfältig untersucht. ${ }^{40}$

9 Mit dem Blick auf die Kommunikation zwischen der Elite und den einfacheren Bürger gewannen auch andere Begegnungsstätten an Bedeutung. Egon Flaig hat betont, dass die Aktualisierung von Passagen aus Theaterstücken durch die versammelten Zuschauer als politische Meinungsäußerung bedeutsam war, ${ }^{41}$ zudem hat er sich mit der pompa funebris beschäftigt. ${ }^{42}$ Hans Beck und umfänglich Karl-Joachim Hölkeskamp haben den sog. "spatial turn" aufgegriffen und die verschiedenen Arten von Prozessionen analysiert und mit der Erinnerungslandschaft in Rom verknüpft. ${ }^{43}$ Breit hat sich vor allem auch Uwe Walter der römischen Erinnerungskultur und den konkreten Formen des Umgangs mit der Vergangenheit gewidmet. ${ }^{44}$ Die Orientierung auf den mos maiorum ist $u$. a. von Wolfgang Blösel und Michael Stemmler untersucht worden, das Problem der Abweichungstoleranz ist von Jani Kirov behandelt worden. ${ }^{45}$ Die Censur mit ihrer besonderen Verpflichtung auf die Wahrung der Tradition hat Ernst Baltrusch untersucht; Rene Pfeilschifter hat den Census als Integrationsritual behandelt und aus dieser Perspektive verständlich machen können, warum der Census schon vor dem Ende der Republik mehr oder weniger abstarb: Durch die gelockerten Vertretungsregeln waren die Angehörigen der Führungsschicht nicht mehr präsent, sondern schickten ihre Verwalter, so dass hier nicht mehr das ganze Volk erschien und Gemeinschaftsgefühle entwickeln konnte. ${ }^{46}$ Dem römischen Triumph hat Tanja Itgenshorst eine gründliche Analyse unter Konzentration auf die republikanischen Quellen gewidmet und die rituelle Struktur erhellt..77 
102004 publizierte Karl-Joachim Hölkeskamp sein Buch Rekonstruktionen einer Republik. Die politische Kultur des antiken Rom und die Forschungen der letzten Jahrzehnte, das schon 2008 ins Französische, 2010 erheblich erweitert ins Englische, 2016 auch ins Italienische und 2019 ins Spanische übersetzt wurde. Schon allein diese Ausgaben in all den Sprachen, in denen die Altertumswissenschaften üblicherweise kommunizieren, zeigen den enormen Erfolg dieses neuen Meilensteins der Forschung, der eine umfassende Bestandsaufnahme bietet und gleichzeitig klar herausarbeitet, wie fruchtbar die Konzepte der politischen Kulturforschung für das Verständnis der römischen Republik gewesen sind. Seine Ansätze hat Hölkeskamp seither in programmatischen Artikeln etwa $\mathrm{zu}$ den performativen und räumlichen Komponenten der Prozessionen ${ }^{48}$ und $\mathrm{zu}$ Konkurrenz und Agonalität ${ }^{49}$ weiter vertieft. Dem senatorischen Konkurrenzraum und seinem Zusammenbruch am Ende der Republik hat Marian Nebelin eine begrifflich und analytisch eindrucksvolle Untersuchung gewidmet. ${ }^{50}$

11 Christian Meiers bahnbrechendes Werk Respublica amissa hat aber nicht nur die Forschung inspiriert, neu und intensiv darüber nachzudenken, wie und warum denn die Republik funktionierte, sondern vor allem auch darüber, inwieweit und warum sie nicht funktionierte - zumindest in ihren letzten Jahren. In der neuen Einleitung zur 2. Auflage des Werkes aus dem Jahre 1980, die hier in gekürzter Form in französischer Übersetzung vorgelegt wird, hat Meier noch einmal neue Impulse gegeben, denn in seiner erneuten Reflexion wurde manches theoretisch stärker durchdrungen, was im Originalwerk eher deskriptiv notiert worden war. 2015 hat Meier zudem seine Vorstellungen von der "Ordnung der römischen Republik» ausführlich zusammengefasst. ${ }^{51}$ Seine eingehenden Forschungen über die Rolle Caesars, die in der umfangreichen Biographie von 1982 gipfelten, ${ }^{52}$ boten neben anderem auch eine Vertiefung der These von der »Krise ohne Alternative«, sah Meier doch bei Caesar, der zweifellos die Stellung eines Alleinherrschers erreichte, eine große Ratlosigkeit, was denn mit der dominierenden Stellung anzufangen sei - letztlich eine Fortsetzung der Alternativlosigkeit der alten Republik. Zudem hatte Meier an der Entwicklung der Theorie historischer Prozesse mitgearbeitet und seine Krise ohne Alternative als autonomen Prozess in einem abstrakteren Konzept verortet.53 Das zeitgenössische Krisenbewusstsein der späten Republik wurde wieder stärker zum Thema. Nachdem Klaus Martin Girardet und Gustav Adolf Lehmann die Reformfähigkeit der Republik verteidigt hatten, ${ }^{54}$ analysierte Meiers Schülerin Monika Bernett das Krisendenken Ciceros, ${ }^{55}$ in einem Tagungsband zum Werk Christian Meiers dokumentierte sie dann die Verwendung des Begriffs der "Krise ohne Alternative« in Meiers Werk. ${ }^{56}$ Aloys Winterling, ebenfalls ein Schüler Meiers, der sich überwiegend mit der Kaiserzeit beschäftigt hat, identifizierte in demselben Band bei Meier drei unterschiedliche Füllungen des Begriffs der Krise, von denen er eine, mit der ein massives Defizitempfinden, auf das mit verzweifelter Steigerung der vertrauten Aktivitäten reagiert wurde, charakterisiert ist, mit dem aus der Ethnologie übernommenen Begriff der »Involution « bezeichnete. ${ }^{57}$ Diese Diagnose ist von Wolfgang Blösel von einer ganz anderen Seite her bestätigt worden. Er hat festgestellt, dass vor allem in der nachsullanischen Republik eine große Anzahl von Consuln und Praetoren die Übernahme einer Provinz ablehnten und damit auf möglichen militärischen Ruhm und materiellen Gewinn verzichteten. ${ }^{58}$ Es scheint eine Vorstellung vorgeherrscht zu haben, dass man sich von dem hektischen Politikbetrieb in Rom nicht länger entfernen könne, ohne an Einfluss einzubüßen. Anders hat Jürgen von Ungern-Sternberg einen nachlassenden Druck vonseiten des Reiches und überhaupt der Außenpolitik 
konstatiert, wodurch die römischen Aristokraten der Pflicht enthoben wurden, sich um der Verteidigung und Ausweitung des Reiches willen immer wieder miteinander zu arrangieren; statt dessen konnten sie sich jetzt ungehindert ihren Machtkämpfen im Inneren widmen und die Belange der einfachen Leute vernachlässigen. ${ }^{59}$ Uwe Walter hat die Kontingenzanteile in den Entwicklungen einer grundsätzlicheren Reflektion unterzogen, ${ }^{60}$ auch hat er unlängst ein wichtiges Überblickswerk verfasst. ${ }^{61}$

Der Einschätzung Christian Meiers, dass Caesars Herrschaft keine Perspektive zur Überwindung der Krise aufweist, ${ }^{62}$ hat Mischa Meier einen neuen Ansatz entgegengesetzt. Mit Hilfe der Unterscheidung von Diskurs und Handlungsrahmen hat er für Caesar konstatiert, im Handlungsrahmen sei die Monarchie, die Überwindung der republikanischen Krise, schon dagewesen, während sie im Diskurs noch lange unsagbar blieb. ${ }^{63}$ Zudem hat Jan Timmer die Kategorie "Vertrauen« in die Debatte um die Krise der Republik eingeführt und deren Verlust als mögliche Komponente im Untergangsszenario diskutiert, wobei es - wie er selbst hervorhebt - angesichts der Quellenlage schwer zu entscheiden ist, ob die Bürgerkriege seit den 40er Jahren des 1. Jh.s v. Chr. stärker Folge oder stärker Ursache des Vertrauensverlustes waren. ${ }^{64}$

13 Nach all den lebendigen Diskussionen in den letzten Jahrzehnten ist die römische Republik weiterhin aktuell in der althistorischen Forschung in Deutschland, wenn auch vielleicht nicht mehr im vergleichbaren Ausmaß wie noch vor einigen Jahren. Dafür gibt es eine Reihe von Werken zur frühen Kaiserzeit, die durchaus auch von Ansätzen der politischen Kulturforschung inspiriert sind. Die Untersuchung von Andreas Klingenberg über den sozialen Abstieg in der Kaiserzeit geht der vermehrten Beschäftigung mit Verlierern in der römischen Republik voraus. ${ }^{65}$ Mit dem römischen Senat der Kaiserzeit beschäftigen sich Isabelle Künzer, die eine spezifische Kultur der Konkurrenz herausarbeitet, und Simone Blochmann, die dezidiert die "politische Kultur im Senat der frühen Kaiserzeit« - so der Untertitel - analysiert. ${ }^{66}$ Die Rolle des Volkes, die ja lange ein prominentes Thema bei der Erforschung der politischen Kultur der Republik gewesen ist, hat Katja Kröss für die Kaiserzeit untersucht. ${ }^{67}$ Wenn man spekuliert, wie sich die Perspektiven und Ansätze weiter entwickeln werden, dann kann man sicher damit rechnen, dass die Verhaltensregeln der römischen Kultur und ihre langsamen Veränderungen weiter von Interesse sein werden, womöglich mit einem verstärkten Bezug auf Arenen, d.h. auf in Räume, Gattungen, Kommunikationsgemeinschaften eingebundene Regelhaftigkeiten, die den Mitgliedern einer Kultur ansozialisiert werden. Damit eng verknüpft werden Normvorstellungen und ihre unterschiedlichen Verbindlichkeiten zweifellos ein Thema bleiben, und die neue Staatlichkeitsdebatte mit ihren interessanten, weil gerade nicht auf den modernen Staat fixierten Kategorien wird für die Antike fruchtbar werden. ${ }^{68}$ Aber letztlich bleibt $\mathrm{zu}$ hoffen, dass sich viel Unerwartetes und daher umso Aufregenderes ereignet - wie es 1966 mit dem Erscheinen von Res publica amissa geschah. 


\section{BIBLIOGRAPHIE}

Alföldy, G. (1975): Römische Sozialgeschichte, Wiesbaden; 2. Aufl. 1979, 3. Aufl. 1984.

Alföldy, G. (2011): Römische Sozialgeschichte, 4. völlig überarbeitete und aktualisierte Auflage, Stuttgart.

Angius, A. (2018): La Repubblica delle opinioni. Informazione politica e partecipazione popolare a Roma tra II e I secolo a.C., Milano.

Baltrusch, E. (1989): Regimen morum. Die Reglementierung des Privatlebens der Senatoren und Ritter in der römischen Republik und frühen Kaiserzeit, München.

Beck, H. (2005): »Züge in die Ewigkeit. Prozessionen durch das republikanische Rom«, Göttinger Forum für Altertumswissenschaft, 8, S. 73-104.

Beck, H. (2019): »Pecuniam inlargibo tibi. Wahlbestechung und Wahlniederlage in der mittleren römischen Republik«, in: Hölkeskamp, K.-J. / Beck, H. (Hg.): Verlierer und Aussteiger in der `Konkurrenz unter Anwesendenı. Agonalität in der politischen Kultur des antiken Rom, Stuttgart, S. 3153.

Bernett, M. (1995): Causarum cognitio. Ciceros Analysen zur politischen Krise der späten römischen Republik (Palingenesia, 31), Stuttgart.

Bernett, M. (2008): »Krisenbewußtsein der späten römischen Republik«, in: Bernett / Nippel / Winterling (2008), S. 161-179.

Bernett, M. / Nippel, W. / Winterling, A. (Hg.) (2008): Christian Meier zur Diskussion. Autorenkolloquium am Zentrum für Interdisziplinäre Forschung in Bielefeld, Stuttgart.

Bleicken, J. (1955): Das Volkstribunat der klassischen Republik. Studien zu seiner Entwicklung zwischen 287 und 133 v.Chr., München; 2. Aufl. 1968).

Bleicken, J. (1975): Lex publica. Gesetz und Recht in der römischen Republik, Berlin / New York.

Blochmann, S. (2017): Verhandeln und entscheiden. Politische Kultur im Senat der frühen Kaiserzeit (Historia Einzelschriften, 245), Stuttgart.

Blösel, W. (2000): „Die Geschichte des Begriffs mos maiorum von den Anfängen bis zu Cicero«, in: Linke, B. / Stemmler, M. (Hg.): Mos maiorum. Untersuchungen zu den Formen der Identitätsstiftung und Stabilisierung in der römischen Republik (Historia Einzelschriften, 141), Stuttgart, S. 25-97.

Blösel, W. (2011): »Die Demilitarisierung der römischen Nobilität von Sulla bis Caesar«, in: Blösel, W. / Hölkeskamp, K.-J. (Hg.): Von der militia equestris zur militia urbana. Prominenzrollen und Karrierefelder im antiken Rom, Beiträge einer internationalen Tagung vom 16. bis 18. Mai an der Universität zu Köln, Stuttgart, S. 55-80.

Blösel, W. (2016): »Provincial Commands and Money in the Late Roman Republic«, in: Beck, H. / Jehne, M. / Serrati, J. (Hg.): Money and Power in the Roman Republic (Collection Ltomus, 355), Brüssel, S. 68-81.

Blösel, W. (in Vorbereitung): Imperia extraordinaria liberae rei publicae - Studien zur Demilitarisierung der römischen Nobilität, Habilitationsschrift Köln.

Bruhns, H. (1978): Caesar und die römische Oberschicht in den Jahren 49-44 v.Chr. Untersuchungen zur Herrschaftsetablierung im Bürgerkrieg (Hypomnemata, 55), Göttingen. 
Bruhns, H. (1997): »Zur Wirtschaftsgeschichte der römischen Republik in der deutschen Forschung. Kommentar zum Beitrag von Jean Andreau«, in: Bruhns / David / Nippel (1997), S. 185-193.

Bruhns, H. (2003): „Crise de la République romaine? Quelle crise?«, in: Franchet d'Espèrey, S. / Fromentin, V. / Gotteland, S. / Roddaz, J.-M. (Hg.): Fondements et crises du pouvoir (AusoniusPublications Études, 9), Bordeaux, S. 365-378.

Bruhns, H. / David, J.-M. / Nippel, W. (Hg.) (1997): Die späte römische Republik - La fin de la République romaine. Un débat franco-allemand d'histoire et d'historiographie (Collection de l'École française de Rome, 235), Paris / Rom.

Burckhardt, L. A. (1990): »The Political Elite oft he Roman Republic: Comments on Recent Discussion of the Concepts nobilitas and homo novus«, Historia, 39, S. 77-99.

Degelmann, C. (2018): Squalor. Symbolisches Trauern in der Politischen Kommunikation der Römischen Republik und Frühen Kaiserzeit (Potsdamer Altertumswissenschaftliche Beiträge, 61), Stuttgart.

Finley, M. I. (1973): The Ancient Economy, Berkeley / Los Angeles; 2. Aufl. 1984; deutsch: Die antike Wirtschaft, übers. v. A. Wittenburg, München 1977; 3. Aufl. 1993.

Flaig, E. (1992): Den Kaiser herausfordern. Die Usurpation im Römischen Reich, Frankfurt / New York.

Flaig, E. (1993): »Politisierte Lebensführung und ästhetische Kultur. Eine semiotische Untersuchung am römischen Adel«, Historische Anthropologie, 1, S. 193-217.

Flaig, E. (1994): »Repenser le politique dans la République romaine«, Actes de la Recherche en Sciences sociales, 105, S. 13-25.

Flaig, E. (1995a): »Entscheidung und Konsens. Zu den Feldern der politischen Kommunikation zwischen Aristokratie und Plebs«, in: Jehne (1995), S. 77-127.

Flaig, E. (1995b): „Die Pompa Funebris. Adlige Konkurrenz und annalistische Erinnerung in der Römischen Republik«, in: Oexle, O.G. (Hg.): Memoria als Kultur, Göttingen, S. 115-148.

Flaig, E. (1997): »Zwingende Gesten in der römischen Politik«, in: Chvojka, E. / van Dülmen, R. / Jung, V. (Hg.): Neue Blicke. Historische Anthropologie in der Praxis, Wien / Köln / Weimar, S. 33-50.

Flaig, E. (1998): „War die römische Volksversammlung ein Entscheidungsorgan? Institution und soziale Praxis«, in: Blänkner, R. / Jussen, B. (Hg.): Institutionen und Ereignis. Über historische Praktiken und Vorstellungen gesellschaftlichen Ordnens, Göttingen, S. 49-73.

Flaig, E. (2003): Ritualisierte Politik. Zeichen, Gesten und Herrschaft im Alten Rom (Historische Semantik, 1), Göttingen.

Gelzer, M. (1912): Die Nobilität der römischen Republik, Leipzig / Berlin; Nachdruck in: ders. (1962): Kleine Schriften, Band 1, Wiesbaden, S. 17-135.

Girardet, K. M. (1983): Die Ordnung der Welt. Ein Beitrag zur philosophischen und politischen Interpretation von Ciceros Schrift de legibus (Historia Einzelschriften, 42), Wiesbaden.

Girardet, K. M. (2018): »Hat Cicero, ohne es zu bemerken, die Einführung der Monarchie in Rom vorgeschlagen?«, Hermes, 146, S. 116-121.

Harders, A.-C. (2008): Suavissima Soror. Untersuchungen zu den Bruder-Schwester-Beziehungen in der römischen Republik (Vestigia, 60), München.

Heuß, A. (1956): »Der Untergang der römischen Republik und das Problem der Revolution«, Historische Zeitschrift, 182, S. 1-28. 
Heuß, A. (1973): »Das Revolutionsproblem im Spiegel der antiken Geschichte«, Historische Zeitschrift, 216, S. 1-72.

Hölkeskamp, K.-J. (1987): Die Entstehung der Nobilität. Studien zur sozialen und politischen Geschichte der Römischen Republik im 4. Jhdt. v. Chr., Stuttgart; 2. erweiterte Auflage 2011.

Hölkeskamp, K.-J. (1995): »Oratoris maxima scaena. Reden vor dem Volk in der politischen Kultur der Republik«, in: Jehne (1995), S. 11-49;

überarbeiteter und ergänzter Nachdruck in: Hölkeskamp, K.-J. (2004): SENATVS POPVLVSQVE ROMANVS. Die politische Kultur der Republik - Dimensionen und Deutungen, Stuttgart, S. 219-256.

Hölkeskamp, K.-J. (2004): Rekonstruktionen einer Republik. Die politische Kultur des antiken Rom und die Forschung der letzten Jahrzehnte (Historische Zeitschrift Beihefte [N.F.], 38), München; Übersetzungen: französisch 2008; englisch 2010; italienisch 2016; spanisch 2019.

Hölkeskamp, K.-J. (2006): »Konsens und Konkurrenz. Die politische Kultur der römischen Republik in neuer Sicht«, Klio, 88, S. 360-396; überarbeiteter Nachdruck in: Hölkeskamp, K.-J. (2017): LIBERA RES PUBLICA. Die politische Kultur des antiken Rom-Positionen und Perspektiven, Stuttgart, S. 123-161.

Hölkeskamp, K.-J. (2008): »Hierarchie und Konsens. Pompae in der politischen Kultur der römischen Republik«, in: Arweiler, A. H. / Gauly, B. M. (Hg.): Machtfragen. Zur kulturellen Repräsentation und Konstruktion von Macht in Antike, Mittelalter und Neuzeit, Stuttgart, S. 79-126; überarbeiteter Nachdruck in: Hölkeskamp, K.-J. (2017): LIBERA RES PUBLICA. Die politische Kultur des antiken Rom - Positionen und Perspektiven, Stuttgart, S. 189-236.

Hölkeskamp, K.-J. (2011): „Self-serving Sermons. Oratory and the self-construction of the republican aristocrat «, in: Smith, C. / Corvino, R. (Hg.): Praise and Blame in Roman Republican Rhetoric, Swansea, S. 17-34.

Hölkeskamp, K.-J. (2013a): »Friends, Romans, countrymen. Addressing the Roman People and the Rhetoric of Inclusion «, in: Steel, C. / van der Blom, H. (Hg.): Community and Communication. Oratory and Politics in Republican Rome, Oxford, S. 11-28.

Hölkeskamp, K.-J. (2013b): »Concordia contionalis. Die rhetorische Konstruktion von Konsens in der römischen Republik«, in: Flaig, E. / Müller-Luckner, E. (Hg.): Genesis und Dynamiken der Mehrheitsentscheidung, München, S. 101-128;

überarbeiteter Nachdruck in: Hölkeskamp, K.-J. (2017): LIBERA RES PUBLICA. Die politische Kultur des antiken Rom-Positionen und Perspektiven, Stuttgart, S. 163-188.

Hölkeskamp, K.-J. (2014): »Konkurrenz als sozialer Handlungsmodus - Positionen und Perspektiven der historischen Forschung«, in: Jessen, R. (Hg.): Konkurrenz in der Geschichte. Praktiken - Werte - Institutionalisierungen, Frankfurt / New York, S. 33-57.

Hölkeskamp, K.-J. (2015): »>Performative turn` meets `spatial turn`. Prozessionen und andere Rituale in der neueren Forschung", in: Boschung, D. / Hölkeskamp, K.-J. / Sode, C. (Hg.): Raum und Performanz. Rituale in Residenzen von der Antike bis 1815, Stuttgart, S. 15-74.

Hölkeskamp, K.-J. (2017): »Politische Kultur - Karriere eines Konzepts. Ansätze und Anwendungen am Beispiel der römischen Republik«, in: Haake, M. / Harders, A.-C. (Hg.): Politische Kultur und soziale Struktur der römischen Republik. Akten eines Kolloquiums aus Anlass des 70. Todestags von Friedrich Münzer, Stuttgart, S. 457-495; überarbeiteter Nachdruck in: Hölkeskamp, K.-J. (2017): LIBERA RES PUBLICA. Die politische Kultur des antiken Rom -Positionen und Perspektiven, Stuttgart, S. 73-105. 
Hölkeskamp, K.-J. (2019a): »)Cultural Turn oder gar Paradigmenwechsel in der Althistorie? Die politische Kultur der römischen Republik in der neueren Forschung «, Historische Zeitschrift, 309, S. 1-35.

Hölkeskamp, K.-J. (2019b): »Verlierer in der `Konkurrenz unter Anwesenden`. Agonalität in der politischen Kultur der römischen Republik«, in: Hölkeskamp, K.-J. / Beck, H. (Hg.): Verlierer und Aussteiger in der `Konkurrenz unter Anwesenden‘. Agonalität in der politischen Kultur des antiken Rom, Stuttgart, S. 11-29.

Itgenshorst, T. (2005): Tota illa pompa. Der Triumph in der römischen Republik, Göttingen.

Jehne, M. (1987): Der Staat des Dictators Caesar, Köln / Wien.

Jehne M. (Hg.) (2015): Demokratie in Rom? Die Rolle des Volkes in der Politik der römischen Republik (Historia Einzelschriften, 96), Stuttgart.

Jehne, M. (1995a): »Einführung: Zur Debatte um die Rolle des Volkes in der römischen Politik«, in: Jehne (1995), S. 1-9.

Jehne, M. (1995b): „Die Beeinflussung von Entscheidungen durch >Bestechung`: Zur Funktion des ambitus in der römischen Republik«, in: Jehne (1995), S. 51-76.

Jehne, M. (2000): »Jovialität und Freiheit. Zur Institutionalität der Beziehungen zwischen Oberund Unterschichten in der römischen Republik«, in: Linke, B. / Stemmler, M. (Hg.): Mos maiorum. Untersuchungen zu den Formen der Identitätsstiftung und Stabilisierung in der römischen Republik (Historia Einzelschriften, 141), Stuttgart, S. 207-235.

Jehne, M. (2001/2003a): »Integrationsrituale in der römischen Republik. Zur einbindenden Wirkung der Volksversammlungen«, in: Urso, G. (Hg.): Integrazione, mescolanza, rifiuto. Incontri di popoli, lingue e culture in Europa dall'Antichità all'Umanesimo, Atti del convegno internazionale, Cividale del Friuli, 21-23 settembre 2000, Roma 2001, S. 89-113; leicht überarbeitet in: Hölkeskamp, K.-J. / Rüsen, J. / Stein-Hölkeskamp, E. / Grütter, H. Th. (Hg.) (2003): Sinn (in) der Antike. Orientierungssysteme, Leitbilder und Wertkonzepte im Altertum, Mainz, S. 279-297.

Jehne, M. (2003b): »Krisenwahrnehmung und Vorschläge zur Krisenüberwindung bei Cicero«, in: Franchet d'Espèrey, S. / Fromentin, V. / Gotteland, S. / Roddaz, J.-M. (Hg.): Fondements et crises du pouvoir (Ausonius-Publications Études, 9), Bordeaux, S. 379-396.

Jehne, M. (2006a): »Who Attended Roman Assemblies? Some Remarks on Political Participation in the Roman Republic«, in: Marco Simón, F. / Pina Polo, F. / Remesal Rodríguez, J. (Hg.): Repúblicas y ciudadanos: modelos de participación cívica en el mundo antiguo (Instrumenta, 21), Barcelona, S. 221234.

Jehne, M. (2006b): »Methods, Models, and Historiography«, in: Rosenstein, N. / Morstein-Marx, R. (Hg.): A Companion to the Roman Republic, Oxford, S. 3-28.

Jehne, M. (2008): »Christian Meier und Iulius Caesar, oder: Das Faszinosum des Außenseiters«, in: Bernett / Nippel / Winterling (2008), S. 201-217.

Jehne, M. (2009a): „Caesars Alternative(n). Das Ende der römischen Republik zwischen autonomem Prozeß und Betriebsunfall«, in: Hölkeskamp, K.-J. (Hg.): Eine politische Kultur (in) der Krise? Die »letzte Generation « der römischen Republik (Schriften des Historischen Kollegs, 73), München, S. 141-160.

Jehne, M. (2009b): »Le système électoral des Romains et le désespoir des candidats«, RHD, 87, S. 498-501. 
Jehne, M. (2010): „Die Dominanz des Vorgangs über den Ausgang. Struktur und Verlauf der Wahlen in der römischen Republik«, in: Dartmann, Chr. / Wassilowsky, G. / Weller, Th. (Hg.): Technik und Symbolik vormoderner Wahlverfahren (Historische Zeitschrift Beiheft, 52), München, S. 17-34.

Jehne, M. (2011): »Blaming the people in front of the people. Restraint and outbursts of orators in Roman contiones«, in: Smith, Chr. / Covino, R. (Hg.): Praise and Blame in Roman Republican Rhetoric, Swansea, S. 111-125.

Jehne, M. (2013a): »Feeding the plebs with words. The significance of senatorial public oratory in the small world of Roman politics«, in: Steel, C. / van der Blom, H. (Hg.): Community and Communication. Oratory and Politics in Republican Rome, Oxford, S. 49-62.

Jehne, M. (2013b): »Politische Partizipation in der römischen Republik«, in: Reinau, H. / UngernSternberg, J. v. (Hg.): Politische Partizipation. Idee und Wirklichkeit von der Antike bis in die Gegenwart (Colloquium Rauricum, 13), Berlin / Boston, S. 103-144.

Jehne, M. (2020): »Die Chance, eine Alternative zu formulieren, und die Chance, eine Alternative zu verwirklichen. Das Sagbare und das Machbare im republikanischen und augusteischen Rom«, in: Nebelin, M. / Tiersch, C. (Hg.): Semantische Kämpfe zwischen Republik und Prinzipat? Kontinuität und Transformation der politischen Sprache in Rom (Historische Semantik, 31), Göttingen, S. 73-101 (im Druck).

Karataş, S. (2019): Zwischen Bitten und Bestechen. Ambitus in der politischen Kultur der römischen Republik - Der Fall des Cn. Plancius (Hermes Einzelschriften, 115), Stuttgart.

Kirov, J. (2010): »Der Umgang mit Abweichungen in der römischen Republik«, Historische Zeitschrift, 290, S. 297-320.

Klingenberg, A. (2011): Sozialer Abstieg in der römischen Kaiserzeit. Risiken in der Oberschicht in der Zeit von Augustus bis zum Ende der Severer, Paderborn.

Knopf, F. (2018): Die Partizipationsmotive der plebs urbana im spätrepublikanischen Rom, Berlin.

Kröss, K. (2017): Die politische Rolle der stadtrömischen Plebs in der Kaiserzeit( Impact of Empire, 24), Leiden / Boston.

Künzer, I. (2016): Kulturen der Konkurrenz. Untersuchungen zu einem senatorischen Interaktionsmodell an der Wende vom ersten zum zweiten Jahrhundert n.Chr. (Antiquitas I, 68), Bonn.

Laser, G. (1997): Populo et scaenae serviendum est. Die Bedeutung der städtischen Masse in der Späten Römischen Republik, Trier.

Lehmann, G. A. (1980): Politische Reformvorschläge in der Krise der späten römischen Republik. Cicero De legibus III und Sallusts Sendschreiben an Caesar, Meisenheim a. Glan.

Linke, B. (1998): »Die agnatio. Ein römischer Sonderweg in der sozialen Organisation«, Historische Anthropologie, 6, S. 104-131.

Linke, B. (2006): »Bürger ohne Staat? Die Integration der Landbevölkerung in der römischen Republik«, in: Jehne, M. / Pfeilschifter, R. (Hg.): Herrschaft ohne Integration? Rom und Italien in republikanischer Zeit, Frankfurt a. M., S. 65-94.

Linke, B. (2014): »Die Väter und der Staat. Die Grundlagen der aggressiven Subsidiarität in der römischen Gesellschaft«, in: Lundgreen (2014), S. 65-90.

Lundgreen, C. (2011): Regelkonflikte in der römischen Republik. Geltung und Gewichtung von Normen in politischen Entscheidungsprozessen (Historia Einzelschriften, 221), Stuttgart. 
Lundgreen, C. (2014): „Staatsdiskurse in Rom? Staatlichkeit als analytische Kategorie für die römische Republik«, in: ders. (Hg.): Staatlichkeit in Rom?, Stuttgart, S. 15-61.

Lundgreen, C. (Hg.) (2014): Staatlichkeit in Rom? Diskurse und Praxis (in) der römischen Republik, Staatsdiskurse 28, Stuttgart.

Martin, J. (1982): »Das Institut für Historische Anthropologie«, Saeculum, 33, S. 375-380; wieder abgedruckt in: Martin (2009), S. 199-204.

Martin, J. (1994): »Der Wandel des Beständigen. Überlegungen zu einer historischen Anthropologie«, Freiburger Universitätsblätter, 33 Heft 126, S. 35-46;

wieder abgedruckt in: Winterling, A. (Hg.) (2006): Historische Anthropologie. Basistexte, Stuttgart, S. 143-157; außerdem in Martin (2009), S. 205-219.

Martin, J. (2009): Bedingungen menschlichen Handelns in der Antike. Gesammelte Beiträge zur Historischen Anthropologie, Stuttgart.

Meier, C. (1966): Res publica amissa. Eine Studie zu Verfassung und Geschichte der späten römischen Republik, Stuttgart; 2. Aufl. Frankfurt am Main, 1980.

Meier, C. (1978): »Fragen und Thesen zu einer Theorie historischer Prozesse«, in: Faber, K.-G. / Meier, C. (Hg.): Historische Prozesse, München, S. 11-66.

Meier, C. (1982): Caesar, Berlin.

Meier, C. (2008): »Antworten«, in: Bernett / Nippel / Winterling (2008), S. 259-310.

Meier, C. (2015): »Die Ordnung der Römischen Republik«, Historische Zeitschrift, 300, S. 593-697.

Meier, M. (2014): Caesar und das Problem der Monarchie in Rom (Schriften der Philosophischhistorischen Klasse der Heidelberger Akademie der Wissenschaften, 52), Heidelberg.

Meyer, I. (2006): Von der Vision zur Reform. Der Staat der Gesetze: Ciceros Programm einer Neuordnung der Römischen Republik, 56 - 51 v.Chr. (Quellen und Forschungen zur Antiken Welt, 50), München.

Millar, F. (1977): The Emperor in the Roman World (31 BC-AD 337), London; 2. Aufl. 1992.

Millar, F. (1998): The Crowd in Rome in the Late Republic, Ann Arbor.

Millar, F. (2002): Rome, the Greek World, and the East, Volume 1: The Roman Republic and the Augustan Revolution, Chapel Hill / London.

Mommsen, Th. (1976): Römische Geschichte, Bände 1-3 und 5, Nachdruck der 9. Auflage (19021904) in 8 Bänden, München.

Mommsen, Th. (1887/88): Römisches Staatsrecht, 3. Aufl., 3 Bände in 5, Leipzig.

Mouritsen, H. (2001): Plebs and Politics in the Late Roman Republic, Cambridge.

Mouritsen, H. (2017): Politics in the Roman Republic, Cambridge.

Nebelin, M. (2014): »Aristokratische Konkurrenz in der späten römischen Republik.

Möglichkeitsraum - Soziale Schließung - Transformation«, in: Jessen, R. (Hg.): Konkurrenz in der Geschichte. Praktiken - Werte - Institutionalisierungen, Frankfurt / New York, S. 141-174.

Nebelin, M. (in Vorbereitung): Freiheit und Gewalt. Die Semantik des Politischen bei Cicero, Diss. Dresden.

Nicolet, C. (1976): Le métier de citoyen dans la Rome républicaine, Paris, 2. Aufl. 1979.

Nicolet, C. (1980): The World of the Citizen in Republican Rome, übers. v. P. S. Falla, Berkeley.

Nippel, W. (1988): Aufruhr und »Polizei« in der römischen Republik, Stuttgart. 
Nippel, W. (1995): Public Order in Ancient Rome, Cambridge.

Pfeilschifter, R. (2002): »Die Brüchigkeit der Rituale. Bemerkungen zum Niedergang der römischen Zensur«, Klio, 84, S. 440-464.

Pina Polo, F. (1996): Contra arma verbis. Der Redner vor dem Volk in der späten römischen Republik (HABES, 22), Stuttgart.

Raaflaub, K. (1974): Dignitatis contentio. Studien zur Motivation und politischen Taktik im Bürgerkrieg zwischen Caesar und Pompeius (Vestigia, 20), München.

Rilinger, R. (1976): Die Rolle des Wahlleiters bei den römischen Konsulwahlen von 366 bis 50 v.Chr. (Vestigia, 24), München.

Rilinger, R. (1982): „Die Interpretation des Niedergangs der römischen Republik durch >Revolution` und `Krise ohne Alternative«", Archiv für Kulturgeschichte, 64, S. 279-306; Nachdruck in: Rilinger, R. (2007): Ordo und dignitas. Beiträge zur römischen Verfassungs- und Sozialgeschichte, Stuttgart, S. 123-150.

Rosillo-López, C. (2017): Public Opinion and Politics in the Late Roman Republic, Cambridge.

Samotta, I. (2009): Das Vorbild der Vergangenheit. Geschichtsbild und Reformvorschläge bei Cicero und Sallust (Historia Einzelschriften, 204), Stuttgart.

Schmitz, W. (2009): »Vorwort«, in: Martin (2009), S. 7-16.

Schneider, H. (1974): Wirtschaft und Politik. Untersuchungen zur Geschichte der späten römischen Republik, Erlangen.

Schneider, H. (1977): Die Entstehung der römischen Militärdiktatur. Krise und Niedergang einer antiken Republik, Köln.

Stemmler, M. (2000): »Auctoritas exempli. Zur Wechselwirkung von kanonisierten Vergangenheitsbildern und gesellschaftlicher Gegenwart in der spätrepublikanischen Rhetorik«, in: Linke, B. / Stemmler, M. (Hg.): Mos maiorum. Untersuchungen zu den Formen der Identitätsstiftung und Stabilisierung in der römischen Republik (Historia Einzelschriften, 141), Stuttgart, S. 141-205.

Strasburger, H. (1931): Concordia Ordinum. Eine Untersuchung zur Politik Ciceros, Diss. Frankfurt a.M., Borna-Leipzig.

Strasburger, H. (1953): „Caesar im Urteil seiner Zeitgenossen«, Historische Zeitschrift, 175, S. 225264.

Strasburger, H. (1968): Caesar im Urteil seiner Zeitgenossen, 2. Aufl., Darmstadt.

Tiersch, C. (2009): »Politische Öffentlichkeit statt Mitbestimmung? Zur Rolle der contiones in der mittleren und späten römischen Republik«, Klio, 91, S. 40-68.

Timmer, J. (2009): »Auseinandertreten, wenn alle einer Meinung sind - Überlegungen zur discessio«, Klio, 91, S. 384-405.

Timmer, J. (2017): Vertrauen. Eine Ressource im politischen System der römischen Republik, Frankfurt / New York.

Tröster, M. (2013): »Roman Politics and the Whims of the Crowd: the Plebs Contionalis Revisited«, Latomus, 72, S. 128-134.

Ungern-Sternberg, J. von (1998): »Die Legitimitätskrise der römischen Republik«, Historische Zeitschrift, 266, S. 607-624. 
Veyne, P. (1976): Le pain et le cirque. Sociologie historique d'un pluralisme politique, Paris; dt. Übers.: Brot und Spiele. Gessellschaftliche Macht und politische Herrschaft in der Antike, übers. v. K. Laermann u. H. R. Brittnacher (Anmerkungen), Frankfurt / New York / Paris, 1988.

Walter, U. (2003): »Ahn Macht Sinn. Familientradition und Familienprofil im republikanischen Rom«, in: Hölkeskamp, K.-J. /Rüsen, J. / Stein-Hölkeskamp, E. / Grütter, H. Th. (Hg.): Sinn (in) der Antike. Orientierungssysteme, Leitbilder und Wertkonzepte im Altertum, Mainz, S. 255-278.

Walter, U. (2004a): Memoria und res publica. Zur Geschichtskultur im republikanischen Rom, Frankfurt a.M.

Walter, U. (2004b): »Ein Ebenbild des Vaters«. Familiale Wiederholungen in der historiographischen Traditionsbildung der römischen Republik«, Hermes, 132, S. 406-425.

Walter, U. (2010): »Patronale Wohltaten oder kriminelle Mobilisierung? Sanktionen gegen unerlaubte Wahlwerbung im spätrepublikanischen Rom«, in: Grüne, N. / Slanicka, S. (Hg.): Korruption. Historische Annäherung an eine Grundfigur politischer Kommunikation, Göttingen, S. 145166.

Walter, U. (2009): „Struktur, Zufall, Kontingenz? Überlegungen zum Ende der römischen Republik«, in: Hölkeskamp, K.-J. / Müller-Luckner, E. (Hg.): Eine politische Kultur (in) der Krise? Die letzte Generation der römischen Republik, München, S. 27-51.

Walter, U. (2017): Politische Ordnung in der römischen Republik, Enzyklopädie der griechisch-römischen Antike, München.

Winterling, A. (2008): »)Krise ohne Alternative im Alten Rom«, in: Bernett / Nippel / Winterling (2008), S. 219-239.

\section{NOTES}

1. C. Meier (1966) (2. Aufl. 1980). Dem Charakter dieses Berichts über die deutsche bzw. deutschsprachige Forschung entsprechend verzichte ich weitestgehend auf eine Dokumentation der Forschungen in anderen Ländern.

2. Mommsen (1976), (1887/88); Gelzer (1912) (Nachdruck 1962); Strasburger (1931), (1953)

(2. Aufl. 1968); zu Heuß unten Anm. 4.

3. Zur Bedeutung gerade von Mommsen und Gelzer für die Erforschung der römischen Republik allgemein vgl. knapp Jehne (2006b), S. 4-6.

4. Vgl. Heuß (1956), (1973).

5. Raaflaub (1974); Bruhns (1978).

6. Rilinger (1976).

7. Rilinger (1982).

8. Bruhns (2003).

9. Nippel (1988); vgl. auch eine darauf aufbauende englische Version 1995.

10. Hölkeskamp (1987) (2. Aufl. 2011). Hölkeskamp ist nicht im formalen Sinne ein Schüler Meiers: Er hat zwar u. a. bei Meier studiert, aber bei Karl-Wilhelm Welwei in Bochum promoviert.

11. Vgl. dazu Martin (2009) (zuerst 1982); dazu auch Schmitz (2009), S. 9-10.

12. So die brillante Formel von Martin (1994).

13. Vgl. z. B. Linke (1998), (2006), (2014); Harders (2010).

14. Vgl. für die Aktivitäten der auslaufenden Arbeitsstelle die Website der Akademie http:// www.sklaven.adwmainz.de/index.php?id=312.

15. Alföldy (1976) (2. Aufl. 1979 u. 3. Aufl. 1984), (2011). 
16. Finley (1973), (1984) (die erste englische Fassung und die erweiterte). Eine deutsche Übersetzung erschien schon 1977 als preiswertes Taschenbuch, in der dritten Auflage (1993) wurde die erweiterte Fassung vorgelegt.

17. Schneider (1974); vgl. auch seine Darstellung des Untergangs der römischen Republik, Schneider (1977). Zur Entwicklung der Wirtschaftsgeschichte Roms in Deutschland vgl. Bruhns (1997).

18. Bleicken (1955) (2. Aufl. 1968).

19. Bleicken (1975).

20. C. Meier (2015), S 599, Anm. 11.

21. Lundgreen (2011). S. auch Lundgreen (2014).

22. Nicolet (1976) (2. Aufl. 1979; amerikanische Fassung 1980); Veyne (1976) (gekürzt auf Deutsch 1988).

23. Cf. Jehne (2006b), S. 12-13.

24. Bruhns / David / Nippel (1997).

25. Millar (1977).

26. Millars Aufsätze zur Republik (zuerst erschienen zwischen 1984 und 1995) sind zusammengestellt in Millar (2002), »Part II: The Roman Republic«. Siehe auch Millar (1998).

27. Vgl. für einen knappen Überblick Jehne (1995a).

28. Jehne (Hg.) (1995).

29. Es gab aber auch schon früher Auseinandersetzungen mit Millars Ideen, vgl. z. B. Burckhardt (1990); Flaig (1992), (1993), (1994); Hölkeskamp (1987).

30. Flaig (1992), S. 84-86. Vgl. schon Flaig (1998).

31. Zu den Wahlen vgl. Jehne (2001/2003a), (2009b), (2010), (2013b).

32. Vgl. außerdem Hölkeskamp (2004), (2017), (2019a).

33. Flaig (2003); siehe auch Flaig (1997).

34. Degelmann (2018).

35. Timmer (2009).

36. Hölkeskamp (1995), (2011), (2013a), (2013b); Jehne (2000), (2001/2003a), (2011), (2013b); Flaig (1995a), (2003); Pina Polo (1996); Knopf (2018).

37. Mouritsen (2001), (2017).

38. Jehne (2006b), (2011), (2013); Hölkeskamp (1995), (2006), (2013a), (2013b).

39. Laser (1997); Tiersch (2009); Tröster (2013); Knopf (2018); in diese Richtung führt auch die neuere Forschung zur öffentlichen Meinung in Rom, vgl. jetzt Rosillo-López (2017); Angius (2018). 40. Vgl. Jehne (2001/2003b), (2009b), (2010), (2013b). Zum ambitus Jehne (1995); Walter (2010); Beck (2019); Karataş (2019).

41. Flaig (1995a), (2003), S. 155-231.

42. Flaig (1995b), (2003), S. 49-68.

43. Hölkeskamp (2004), (2008), (2015); vgl. auch Beck (2005).

44. Walter (2004a); Walter (2003); (2004b) (in dieser Trivium-Ausgabe in gekürzter Form ins Französische übertragen).

45. Blösel (2000); Stemmler (2000); Kirov (2010).

46. Baltrusch (1989); Pfeilschifter (2002).

47. Itgenshorst (2005).

48. Hölkeskamp (2015).

49. Hölkeskamp (2014), (2019b).

50. Nebelin (2014).

51. C. Meier (2015).

52. C. Meier (1982).

53. C. Meier (1978). 
54. Durch Analyse der zentralen Texte von Cicero (de legibus und de re publica) und Sallust (epistulae ad Caesarem): vgl. Lehmann (1980); Girardet (1983); später dann Meyer (2006); Samotta (2009).

55. Bernett (1994). S. auch Jehne (2003b), dagegen jetzt Girardet (2018). Leider noch nicht publiziert ist die Dissertation von Marian Nebelin, der die Radikalisierung in Ciceros Denken bis zur Rechtfertigung der gnadenlosen Ausmerzung aller Gegner überzeugend vorgeführt hat (Nebelin [in Vorbereitung]).

56. Bernett (2008). In dem Band hat C. Meier (2008) auf alle Beiträge zu seinen Themen und Werken geantwortet.

57. Winterling (2008).

58. Blösel (2011), (2016). Leider liegt die umfassende Analyse Blösels noch nicht vor (Blösel [in Vorbereitung]).

59. Ungern-Sternberg (1998).

60. Walter (2009).

61. Walter (2017).

62. Vgl. dagegen etwa Jehne (2008), (2009a) (unter Rückgriff auf Meiers Konzept der historischen Prozesse; siehe auch schon Jehne [1987]).

63. M. Meier (2014). Vgl. dazu demnächst auch Jehne (2020 [im Druck]).

64. Timmer (2017).

65. Klingenberg (2012).

66. Künzer (2016); Blochmann (2017).

67. Kröss (2017).

68. Vgl. vorläufig Lundgreen (2014).

INDEX

Mots-clés : République romaine, introduction

Schlüsselwörter : römische Republik, Einleitung

\section{AUTEUR}

\section{MARTIN JEHNE}

Martin Jehne ist Professor für Alte Geschichte an der Technischen Universität Dresden. Nähere Informationen finden Sie hier. 